\title{
Aplicación metodología cualitativa para el estudio del impacto de la pandemia COVID-19 en titulaciones de Ciencias de la Salud, Terapia Ocupacional y Fisioterapia.
}

\author{
Paloma Moro Lopez-Menchero ${ }^{a}$, Domingo Palacios-Ceña ${ }^{b}$, Lidiane Lima Florencio ${ }^{c}$, Jorge Perez \\ Corrales $^{\mathrm{d}}$, Javier Gueita Rodriguez ${ }^{\mathrm{e}}$, Rosa $\mathbf{M}^{\mathrm{a}}$ Martinez Piedrola ${ }^{\mathrm{f}}$, Marta Perez de Heredia ${ }^{\mathrm{g}}$ y \\ Carmen Jimenez Antona ${ }^{\text {h }}$
}

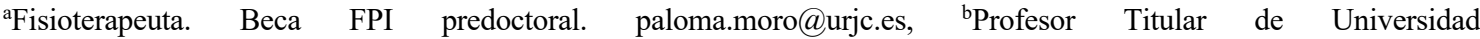
Grupo de Investigación en Humanidades e Investigación Cualitativa en Ciencias de la Salud (Hum\&QRinHS). Universidad Rey Juan Carlos. domingo.palacios@urjc.es, ${ }^{c}$ Profesora Ayudante Doctor. Grupo de investigación de alto rendimiento en Terapia Manual, Punción Seca y Ejercicio Terapéutico (TMPSE). Departamento de Fisioterapia, Terapia Ocupacional, Rehabilitación y Medicina Física. Universidad Rey Juan Carlos. lidiane.florencio@urjc.es, Profesor Ayudante Doctor. Grupo de Investigación en Humanidades e Investigación Cualitativa en Ciencias de la Salud (Hum\&QRinHS). Departamento de Fisioterapia, Terapia Ocupacional, Rehabilitación y Medicina Física. Universidad Rey

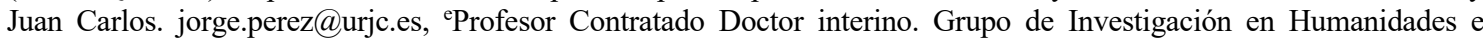
Investigación Cualitativa en Ciencias de la Salud (Hum\&QRinHS). Departamento de Fisioterapia, Terapia Ocupacional, Rehabilitación y Medicina Física. Universidad Rey Juan Carlos. javier.gueita@urjc.es, Profesora Contratado Doctor. Grupo de investigación en Evaluación y Valoración de la Capacidad, funcionalidad y discapacidad (TO+IDI). rosa.martinez@urjc.es, gProfesora Titular de Universidad. Grupo de investigación en Evaluación y Valoración de la Capacidad, funcionalidad y discapacidad (TO+IDI). Departamento de Fisioterapia, Terapia Ocupacional, Rehabilitación y Medicina Física. Universidad Rey Juan Carlos. marta.perezdeheredia@urjc.es, hProfesora Contratado Doctor Grupo de Investigación en Humanidades e Investigación Cualitativa en Ciencias de la Salud (Hum\&QRinHS). Departamento de Fisioterapia, Terapia Ocupacional, Rehabilitación y Medicina Física. Universidad Rey Juan Carlos. carmen.jimenez@urjc.es
\end{abstract}

\section{\$EWMFW}

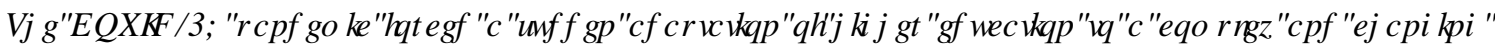

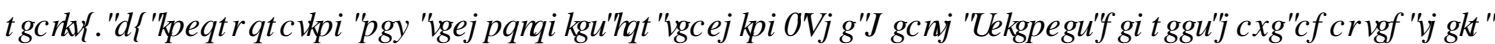

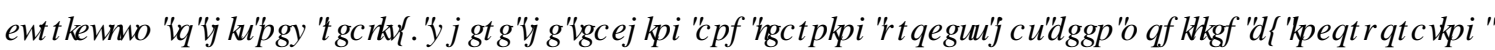

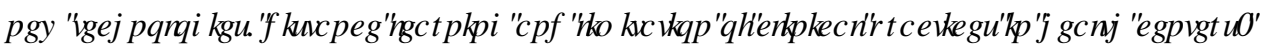

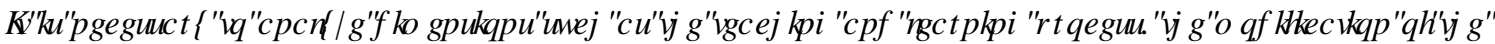

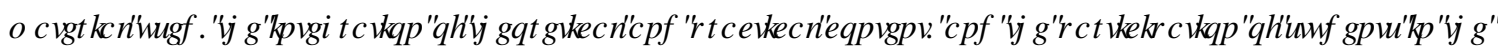
GHHIRSP HQWRILHXFDURQDOP DUAIDO

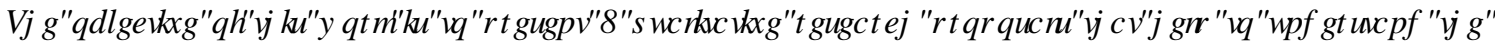

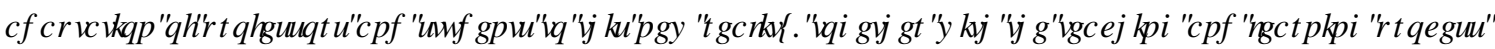

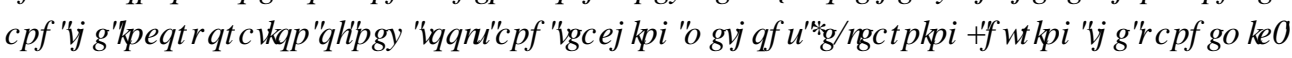

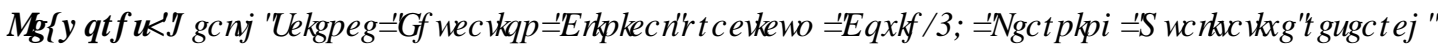

\footnotetext{
5 HXP HQ

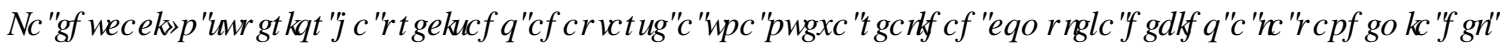

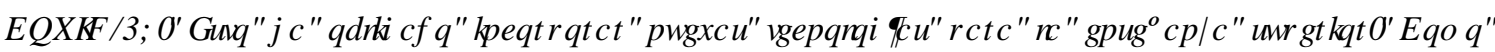

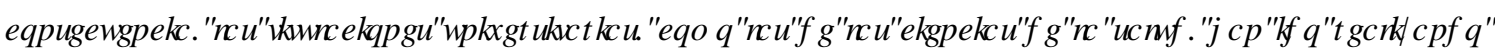

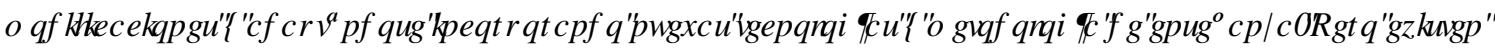

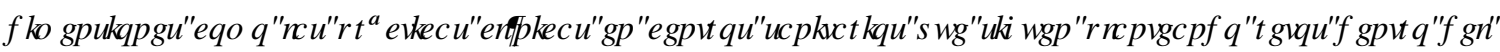
SLRFHRTGHHQMK DQ] DI IDSLHQD] DNAIHQHMDVUWXOFIRQHU]
} 
Aplicación de la metodología cualitativa para el estudio del impacto de la pandemia COVID-19 en titulaciones de ciencias de la salud

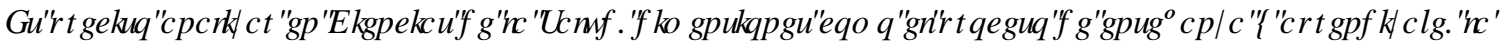

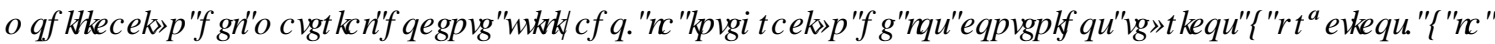

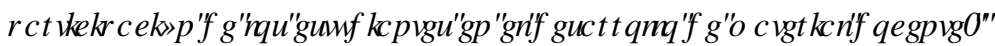

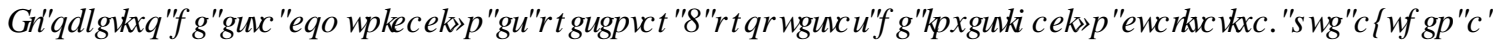

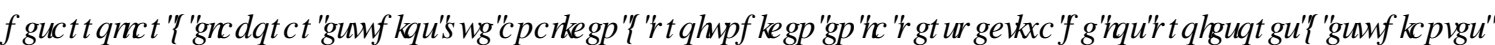

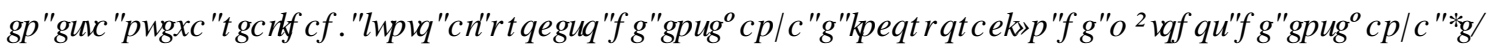

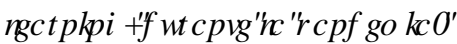

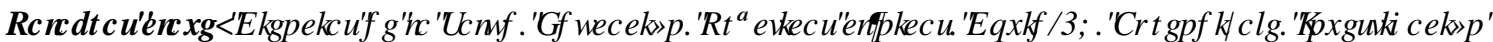
FXDOWDWDDD

\section{Introducción}

La enseñanza de ciencias de la salud (CS) a través de las diferentes disciplinas como enfermería, medicina, fisioterapia, terapia ocupacional y otras, presentan diferencias respecto a otras disciplinas, debido a que sus intervenciones pueden tener efectos iatrogénicos y vulnerar la seguridad de las personas enfermas y las familias (Dickinson y cols., 2020, Patelarouy cols., 2020). Además, aparecen elementos éticos y legales en su práctica que pueden limitar y modificar sus tratamientos e intervenciones (Martins y cols., 2020). Por otro lado, la adquisición de destrezas y competencias técnicas durante las prácticas clínicas y el contacto con entornos clínicos reales forma parte de la base fundamental de su aprendizaje (Mbakaya y cols, 2020).

La pandemia ha provocado una disminución del contacto con entornos clínicos reales en el medio hospitalario y en atención primaria (Garg y cols, 2020, Gómez y cols, 2020), provocando una adaptación de la enseñanza a distancia, a través de e-learning (Sneyd y cols,2020).

Ante este nuevo paradigma es preciso preguntarse: ¿cómo se está aplicando la enseñanza en CS a través de e-learning? ¿En qué se basan los profesores para adaptar sus metodologías en CS en este nuevo entorno? ¿Cómo se van a poder conseguir competencias clínicas adquiridas en entornos clínicos presenciales? ¿Las Tecnologías de Información y Comunicación (TICs) son una opción para adquirir competencias eficaces y seguras en CS?

Para responder a estas preguntas, es necesario describir: a) proceso de enseñanza (qué y cómo se debe enseñar), b) proceso de aprendizaje (qué y cómo se debe aprender), y c) la integración de la práctica clínica real en la enseñanza de CS.

Todas estas preguntas pueden ser contestadas a través de la investigación cualitativa en CS (Tenny y cols., 2020). Esta metodología ayuda a describir la perspectiva de profesores y estudiantes (Dickinson y cols., 2020), muestra obstáculos en los nuevos modelos de innovación docente en entornos clínicos complejos, y a describir el proceso de aprendizaje de estudiantes en entornos clínicos (Mbakaya y cols., 2020).

\section{Objetivos}

Describir propuestas de investigación cualitativa para analizar el proceso de innovación en la docencia en titulaciones de CS, en Terapia Ocupacional y Fisioterapia, durante la pandemia del COVID-19.

Propuesta 1: Estigma percibido en estudiantes de Terapia Ocupacional sobre las personas con trastorno mental grave: estudio cualitativo de casos mediante grupos focales.

Propuesta 2: Adaptación de la prueba ECOE del Grado en Terapia Ocupacional a la modalidad online: estudio cualitativo de casos de la perspectiva de profesores y estudiantes. 
Propuesta 3: Experiencia de la formación académica en estudiantes de Terapia Ocupacional durante la pandemia provocada por la Covid-19: estudio cualitativo mediante DUWEDMHEHHHOFK

Propuesta 4: Experiencias de aprendizaje en las prácticas clínicas hospitalarias (prácticum II) durante la pandemia por Covid-19: Estudio fenomenológico en estudiantes de fisioterapia.

Propuesta 5: Funcionalidad percibida en personas con daño neurológico por parte de los estudiantes de Fisioterapia: estudio cualitativo de casos desde la perspectiva de profesores y estudiantes en la asignatura de Métodos Específicos III de Fisioterapia.

Propuesta 6: Integración de los seminarios de Razonamiento Clínico y Abordajes Específicos en el proceso de estudio de los estudiantes de fisioterapia: Análisis a través de la Teoría Fundamentada durante la pandemia COVID-19 en el curso 2020-21.

\section{Propuestas de investigaciones cualitativas centradas en el proceso de innovación docente en titulaciones de ciencias de la salud, terapia ocupacional y fisioterapia, durante el covid-19}

Las propuestas tienen como objetivo describir el diseño cualitativo fundamental, a través del cual se pueda estudiar y analizar diferentes situaciones y fenómenos. Estas propuestas pueden ser modificadas en función de las necesidades de los profesores/as en función de la titulación, de la asignatura, de las características de los estudiantes, así como del proceso concreto que se quiere describir y/o analizar. Así, estas propuestas son flexibles, para adaptarse al estudio de diferentes $7 R S I F V$.

Las propuestas siguen las recomendaciones internacionales para estudios cualitativos en CS establecidos

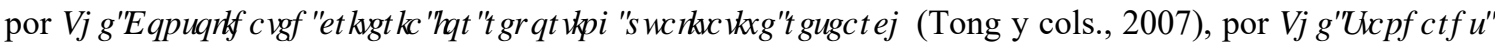

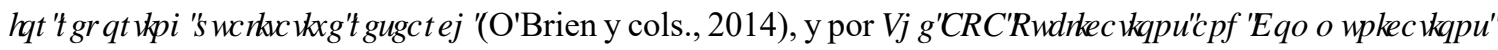
\%RDCFWWIRLFHUSRLW(Levitt y cols., 2018). Estas recomendaciones muestran la estructura que deberían tener los estudios basados en investigación cualitativa, e incluyen como presentar los resultados, y estructura para difundir resultados a la comunidad internacional.

Cada propuesta está formada por subsecciones que fundamentan y orientan la necesidad de investigar el proceso de enseñanza y aprendizaje en durante la pandemia COVID-19 (Korstjens y Moser, 2017), seguido de apartados metodológicos. Todas las propuestas presentadas que guíen cualquier tipo de estudio deberían ser consideradas por un comité ético de investigación clínica (CEIC) antes de ser aplicadas (Creswell y Poth, 2018). El uso y valoración previa del CEIC, es un trámite obligado en CS debido a que se investigará con datos considerados de alta protección, como creencias, comportamientos de salud-enfermedad, sexualidad, etc (Gobierno de España, 1999; Gobierno de España, 2018). La utilización de TICs para investigaciones (grabar audio-voz) durante el confinamiento presenta consideraciones éticas y legales específicas (Archibald y cols., 2019).

\subsection{Propuesta 1. Estigma percibido en estudiantes de Terapia Ocupacional sobre las personas con} trastorno mental grave: estudio cualitativo de casos mediante grupos focales.

\section{पाए \&RQMAR UR}

Las personas con trastorno mental grave (TMG) sufren un alto grado de estigmatización por parte de la sociedad. Los prejuicios sobre esta población también se encuentran presentes entre los profesionales sanitarios. La pandemia ha provocado que la asignatura de Terapia Ocupacional en Salud Mental de $3^{\circ}$ de Grado en Terapia Ocupacional haya funcionado en formato híbrido durante el curso 2020/2021, 


\section{Aplicación de la metodología cualitativa para el estudio del impacto de la pandemia COVID-19 en titulaciones de ciencias de la salud}

impartiéndose la docencia teórica en formato online a través de la plataforma Teams de Microsoft $\mathbb{C}$ y la docencia práctica mediante seminarios prácticos presenciales en grupos reducidos de estudiantes. Además, dentro de los estudiantes que han cursado esta asignatura, hay estudiantes que han realizado sus prácticas clínicas (Practicum I) con esta población y otros estudiantes en contextos clínicos diferentes.

\section{पषण 3 UHXQ⿻}

¿Cómo ha influido el desarrollo de la asignatura de Terapia Ocupacional en Salud Mental en formato híbrido en la percepción del estigma hacia las personas con TMG en estudiantes del Grado en Terapia Ocupacional? ¿Cómo ha influido el desarrollo de la asignatura de Terapia Ocupacional en formato híbrido junto con la realización de prácticas clínicas con esta población en la percepción del estigma hacia las personas con TMG en estudiantes del Grado en Terapia Ocupacional?

\section{पाए 2 ENAMYRV}

a) Describir la influencia de haber cursado la asignatura de Terapia Ocupacional en Salud Mental en la percepción del estigma hacia las personas con TMG en estudiantes del Grado en Terapia Ocupacional; y b) Describir la influencia de haber cursado la asignatura de Terapia Ocupacional en Salud Mental junto con la realización de prácticas clínicas con esta población en la percepción del estigma hacia las personas con TMG en estudiantes del Grado en Terapia Ocupacional.

\section{प्णा ' $\mathrm{LMkR}$}

Estudio cualitativo de casos (Creswell y Poth, 2018) sobre las percepciones, opiniones y creencias de los estudiantes en relación a la percepción del estigma en personas con TMG al finalizar la asignatura y las prácticas clínicas. Este diseño en CS sigue unas recomendaciones específicas mediante el modelo DESCARTE (Carolan y cols., 2016).

\section{पाए 3DUMFLSDQMAR}

Muestreo por propósito no probabilístico (Creswell y Poth, 2018; Moser y Korstjens, 2018) de los estudiantes matriculados en la asignatura de Terapia Ocupacional en Salud Mental perteneciente al tercer curso del Grado en Terapia Ocupacional.

\section{पDW 5HFJIODGHGDRV}

La recogida de datos se realizará mediante una serie de grupos focales a estudiantes que comprenderán, por un lado, aquellos que solamente hayan cursado la asignatura y, por otro, aquellos que hayan cursado la asignatura y además hayan realizado prácticas clínicas con esta población. Además, los investigadores tomarán notas de campo antes, durante y después del desarrollo de los grupos focales sobre los aspectos verbales y no verbales de los participantes.

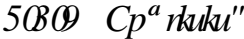

Se aplicará un análisis temático de tipo inductivo (Miles y cols., 2013) de los grupos focales y de las notas de campo mediante triangulación por investigadores.

\subsection{Propuesta 2. Adaptación de la prueba ECOE del Grado en Terapia Ocupacional a la modalidad online: estudio cualitativo de casos de la perspectiva de profesores y estudiantes.}

\section{पाए \&RQM WR}

La prueba ECOE (Evaluación Clínica Objetiva Estructurada) es una prueba de evaluación de competencias clínicas de los estudiantes de Ciencias de la Salud que ha ido sustituyendo de manera progresiva a los tradicionales Trabajos Fin de Grado en los diferentes Grados en Ciencias de la Salud. Debido a la pandemia, 
durante el curso 2019/2020 la prueba ECOE se adaptó del formato presencial a la modalidad online en el Grado de Terapia Ocupacional.

\section{पषण 3UHXQWV}

¿Cómo fue el proceso de adaptación de la prueba ECOE del formato presencial a la modalidad online desde la perspectiva de los profesores? ¿Cómo influyó en la preparación y realización de la prueba ECOE en los estudiantes? ¿Cuáles fueron las barreras y los facilitadores identificados durante su adaptación, preparación y ejecución tanto por los profesores como por los estudiantes?

\section{पाए 2 ENAMRRV}

a) Describir el proceso de adaptación y ejecución de la prueba ECOE a través de la perspectiva de los profesores; b) Describir el proceso de preparación y realización de la prueba ECOE desde la perspectiva de los estudiantes; y c) Describir y analizar barreras y facilitadores de la adaptación, preparación y ejecución desde la perspectiva de los profesores y los estudiantes.

\section{पएव ' LMHR]}

Estudio cualitativo de casos (Yin, 2014) sobre la perspectiva de los profesores y los estudiantes. Este diseño en CS sigue unas recomendaciones específicas mediante el modelo DESCARTE (Carolan y cols., 2016).

\section{प०ए 3DUWFLDQUAR}

Muestreo por propósito no probabilístico (Moser y Korstjens, 2018) de los profesores tanto a tiempo completo como a tiempo parcial que participaron en los procesos de adaptación y ejecución de la prueba ECOE del Grado en Terapia Ocupacional durante el curso 2019/2020, así como de los estudiantes que prepararon y realizaron la prueba ECOE durante el curso 2019/2020.

\section{पDU 5HFRIGDGHODRV}

La recogida de datos se establece en relación al diseño elegido, por lo que se realizarán grupos focales entre los profesores, entre los estudiantes y grupos mixtos formados por profesores y estudiantes. Además, los investigadores tomarán notas de campo antes, durante y después del desarrollo de los grupos focales sobre los aspectos verbales y no verbales de los participantes.

\section{प्पा \$Q QRW}

Se aplicará un análisis temático de tipo inductivo (Creswell y Poth, 2018, Miles y cols., 2013) de los grupos focales y de las notas de campo mediante triangulación por investigadores.

\subsection{Propuesta 3: Experiencia de la formación académica en estudiantes de Terapia Ocupacional durante la pandemia provocada por la Covid-19: estudio cualitativo mediante art-based research.}

\section{पए丁 \&RQMH UR}

Los estudiantes del Grado en Terapia Ocupacional han visto afectado el curso habitual de su formación académica debido a la pandemia desde el mes de marzo de 2020 hasta el momento actual. Durante el segundo semestre del curso 2019/2020 las clases se interrumpieron y comenzaron a impartirse en formato online, teniendo que adaptar también los exámenes a esta modalidad. Durante el curso 2020/2021 las asignaturas funcionaron en formato híbrido, impartiéndose las clases teóricas online y las clases prácticas de manera presencial en grupos reducidos, mientras que los exámenes se realizaron de manera presencial. 
Aplicación de la metodología cualitativa para el estudio del impacto de la pandemia COVID-19 en titulaciones de ciencias de la salud

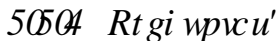

¿Cómo ha sido la experiencia de la formación académica de los estudiantes desde el comienzo de la pandemia hasta el momento actual? ¿Cuál es su perspectiva sobre las adaptaciones realizadas en la metodología docente y evaluadora por parte de los profesores y de la universidad?

\section{पाए 2 ENAMMRV}

a) Describir la experiencia de la formación académica de los estudiantes durante el transcurso de la pandemia; y b) Describir la perspectiva de los estudiantes sobre la influencia de las adaptaciones realizadas en la metodología docente y evaluadora por los profesores y la universidad en su experiencia académica.

\section{प्य ' LMKR]}

Estudio cualitativo mediante art-based research (investigación basada en el arte (Leavy, 2018) que explora la experiencia de los participantes (estudiantes) a través de diferentes medios artísticos.

\section{प⿴囗 3DUMFLDQMAM}

Muestreo por propósito no probabilístico (Moser y Korstjens, 2018) de los estudiantes matriculados en el Grado en Terapia Ocupacional durante los cursos 2019/2020 y 2020/2021.

\section{पTW 5HRJIGDGHODRV}

La recogida de datos se realizará en 2 fases: Primera fase: Acorde con el diseño elegido, la recogida de datos se realizará mediante dibujos o collages realizados por los estudiantes tras un encuadre de la pregunta de investigación realizada por los investigadores (Leavy, 2018). La realización de cada dibujo o collage irá acompañada de un documento narrativo relacionado con el dibujo o collage en el que cada participante explicará su perspectiva y vivencia de la experiencia académica desde el comienzo de la pandemia hasta el momento actual. Segunda fase: Tras analizar los datos de la primera fase, se construirá una entrevista semiestructurada con categorías y preguntas basadas en las experiencias descritas por los participantes y se entrevistará a cada uno de los participantes para profundizar en sus experiencias.

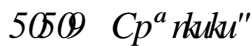

Se aplicará un análisis temático de tipo inductivo (Miles y cols., 2013) de los documentos narrativos aportados por los participantes mediante triangulación por investigadores. Tras las entrevistas se realizará otro análisis temático de tipo inductivo que incluyan tanto los documentos narrativos como las propias entrevistas, mediante triangulación por investigadores.

\subsection{Propuesta 4: Experiencias de aprendizaje en las prácticas clínicas hospitalarias (prácticum II) durante la pandemia por Covid-19: Estudio fenomenológico en estudiantes de fisioterapia.}

\section{पाए \&RQM WR}

Los estudiantes de cuarto en el Grado de Fisioterapia se enfrentan por última vez a las prácticas clínicas en un hospital y han visto afectado el curso habitual de su formación desde marzo 2020 hasta el momento actual. En el Prácticum, aparecen frecuentemente situaciones o eventos críticos que condicionan el aprendizaje, tanto con los pacientes, sus familias o los tutores y compañeros. La pandemia puede acentuar la existencia de esos eventos críticos e influir en el aprendizaje del estudiante. Conociendo que eventos críticos facilitan o dificultan el aprendizaje durante el prácticum en hospitales, se podría evitar o potenciar situaciones que facilitasen el aprendizaje de determinadas competencias. 


\section{प०ए 3 UHXQMD}

¿Cuál es la experiencia de los estudiantes en las prácticas clínicas en hospitales durante la pandemia por Covid-19? ¿Qué eventos/situaciones críticas han marcado su aprendizaje? ¿En qué momento, donde, quien participó, cómo sucedió?

\section{पए丁 2 ENAMIRV}

a) Describir experiencias de estudiantes de $4^{\circ}$ Fisioterapia durante sus últimas prácticas externas hospitalarias, y b) describir eventos críticos durante el aprendizaje en el prácticum II.

\section{पण口 ' $L M k R$}

Estudio cualitativo fenomenológico, orientado a describir y/o analizar la experiencia vivida de los estudiantes durante sus últimas prácticas clínicas (Matua y Van Der Wal, 2015; Wilson, 2015). Este diseño se fundamenta en mostrar la experiencia y perspectiva de las personas que viven esa situación o evento, y utiliza las narraciones en primera persona de los participantes (Matua y Van Der Wal, 2015; Wilson, 2015).

\section{पQT 3DUWFLSDQMAT}

Se realizará un muestreo por propósito de los estudiantes matriculados en la asignatura Prácticum II, de cuarto curso del Grado en Fisioterapia 20-21, y que no tengan asignaturas suspendidas que impidan el acceso a la asignatura Prácticum II. En fenomenología se incluirán aquellas personas que hayan vivido ese fenómeno a estudio, en este caso haber realizado el Prácticum II en hospitales.

\section{पTU 5HFRIGDIGHOWRV}

Se necesita utilizar instrumentos de recogida de datos que se centren en la perspectiva en primera persona de las estudiantes (Matua y Van Der Wal, 2015; Wilson, 2015). Se utilizarán; a) entrevistas en profundidad no-estructuradas, con preguntas abiertas (¿Cuál ha sido su experiencia durante el prácticum II?)

पएव \$Q QMW

Es preciso escoger un sistema de análisis adecuado a la fenomenología. En función de si es fenomenología descriptiva se podría utilizar la propuesta de Giorgi o Gadamer, en caso de ser fenomenología interpretativa se usaría propuestas como la de Van Manen. Lo que caracteriza este tipo de análisis es que es inductivo, no se basa en marco teóricos previos o categorías predefinidas (Matua y Van Der Wal, 2015; Wilson, 2015)

\subsection{Propuesta 5: Funcionalidad percibida en personas con daño neurológico por parte de los estudiantes de Fisioterapia: estudio cualitativo de casos desde la perspectiva de profesores y estudiantes en la asignatura de Métodos Específicos III de Fisioterapia.}

\section{पाए \&RQHA UR}

Las personas con daño neurológico presentan un amplio espectro de signos y síntomas que dificultan su funcionamiento diario. Entenderlo y saberlo valorar es parte fundamental de los fisioterapeutas. Debido a la pandemia surge la necesidad de adaptar la enseñanza online en la asignatura de "Métodos Específicos III de Fisioterapia”. Esta asignatura es fundamental para completar competencias de abordaje especifico, que serán los últimos en su formación académica universitaria.

\section{पषण 3UH XQWV}

¿Cómo ha influido el desarrollo de la asignatura en la percepción de la funcionalidad de los pacientes con daño cerebral en estudiantes del Grado de Fisioterapia? ¿Cómo ha sido el proceso de integración de la enseñanza en la asignatura online con las practicas simuladas? ¿Qué barreras y facilitadores han encontrado los profesores? ¿y los estudiantes? 


\section{पए丁 2 ENAMYRV}

a) Describir el proceso de aprendizaje a través de la perspectiva de los estudiantes y profesoras, y b) describir y analizar barreras y facilitadores de la enseñanza online en la asignatura de Métodos Específicos III.

प⿴囗十 ' LMKR

Estudio cualitativo de casos (Carolan y cols. 2016; Yin, 2014), que incluye diferentes participantes (estudiantes y profesorado).

\section{पПए 3DUKFLDQMAT}

Muestreo por propósito no probabilístico de los estudiantes matriculados en la asignatura en el cuso 2021 , y profesores de la asignatura, tanto de adulto como de infantil.

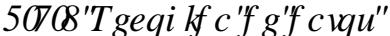

Acorde con el diseño elegido, se utilizarán diversos instrumentos de recogida de datos como, una serie grupos focales al profesorado, una serie de grupos focales a estudiantes, y grupos focales mixtos formados por estudiantes y profesorado.

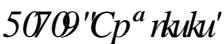

Se aplicará un análisis temático de tipo inductivo para identificar las categorías (Yin, 2014).

3.6 Propuesta 6: Integración de los seminarios de Razonamiento Clínico y Abordajes Específicos en el proceso de estudio de los estudiantes de fisioterapia: Análisis a través de la Teoría Fundamentada durante la pandemia COVID-19 en el curso 2020-21.

\section{पिए \&RQMH UR}

Los alumnos de $4^{\circ}$ de Fisioterapia han visto truncadas muchas experiencias clínicas desde marzo 2020 hasta el momento actual, lo que se ha intentado compensar mediante diversos seminarios de Razonamiento Clínico y Abordajes Específicos con casos reales presentados en video. Esta integración de contenidos teóricos online, y seminarios prácticos, en modalidad presencial o virtual, conocida como "enseñanza híbrida", ha obligado al estudiante de fisioterapia, a integrar la información obtenida por diferentes vías simultáneamente.

\section{पाए 3 UH XQWV}

¿Cómo se integran y coordinan los contenidos online con los seminarios prácticos de casos reales para debatir su razonamiento clínico? ¿Cómo se desarrolla los temas y material de estudio? ¿Cuánto tiempo dedica el estudiante a preparar e integrar esos materiales? ¿Qué barreras y facilitadores han encontrado los estudiantes?

\section{पए丁 2 ENAMYRV}

Describir el proceso de integración del material obtenido vía online durante seminarios específicos con el de las prácticas simuladas presenciales por los estudiantes, e identificar barreras y facilitadores de ese proceso.

\section{पएव ' LMHR]}

Estudio cualitativo basado en la teoría fundamentada (Creswell y Poth, 2018). Este diseño, permite analizar y describir procesos complejos, y confirmar teorías. 


\section{पएव 3DUMFLDQMAM}

Se aplicará un muestreo teórico (Creswell y Poth, 2018), y se reclutarán a estudiantes de fisioterapia matriculados en la asignatura "Métodos Específicos de Fisioterapia III". Este muestreo no probabilístico permite seleccionar a participantes con un perfil basado en su capacidad teórica de aportar información relevante.

\section{पस口प5 HRJIGDGHGDRV}

Se utilizarán entrevistas semiestructuradas, series de grupos focales a cada subgrupo de prácticas de los estudiantes, y observación no participante de los seminarios-prácticos. La observación irá destinada a obtener material realizado por los estudiantes (apuntes, anotaciones in situ), y grabar en vídeo la técnica de fisioterapia por parte del profesorado.

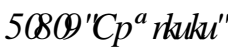

Se aplicará diferentes fases en la codificación; con una primera codificación abierta, seguido de una codificación selectiva y axial. Se aplicará el método de análisis de comparación constante y el uso de memos (Creswell y Poth, 2018).

\section{Resultados.}

De las 6 propuestas presentadas, dos están en fase de reclutamientos (propuestas 1 y 2), dos están siendo valoradas por el comité de ética de investigación clínica - CEIC (propuestas 5 y 6), y dos están en fase de desarrollo (propuestas 3 y 4).

Sobre las propuestas activas ( 1 y 2 ), en la actualidad los investigadores se han encontrado con barreras y dificultades parar reclutar participantes. El COVID-19 ha producido que durante la realización del estudio el contacto directo con potenciales participantes sea limitado. Para controlar esta limitación, se ha incorporado en el protocolo la posibilidad de recoger parte de los datos a través del uso de plataformas digitales tipo Microsoft Teams, Zoom, etc.

Para ello, se ha enviado una modificación al CEIC para incluir una nueva forma de recoger los datos a través de la plataforma virtual Microsoft Teams, y poder realizar la recogida vía online, con posibilidad de grabar audio y vídeo.

Dentro de estas consideraciones se quieren incorporar el siguiente protocolo de reclutamiento:

Primer contacto con los participantes, mediante correo/ teléfono para envío de consentimiento informado y hoja de información, incorporando la posibilidad de realizar la recogida de datos mediante plataforma digital.

Según contacto, donde se confirmar o no su participación, recogida consentimiento informado, y citación del día y hora de la recogida de datos.

Tercer contacto, donde se realiza la recogida de datos. En caso de usar plataforma digital se vuelve a confirmar con la participante su permiso para grabar la recogida de datos, en audio y/o vídeo.

En la actualidad las únicas propuestas en activo son las propuestas 1 y 2 pero están en periodo de pausa, hasta confirmar modificaciones en el protocolo de investigación por el CEIC.

\section{Discusión}

La flexibilidad de los diseños cualitativos permite describir y analizar en profundidad dimensiones subjetivas como los comportamientos, creencias, y valores que incluyen en la toma de decisiones y aceptación de nuevos paradigmas de enseñanza y aprendizaje (Mukhtar y cols., 2020, Khurshid y cols, 


\section{Aplicación de la metodología cualitativa para el estudio del impacto de la pandemia COVID-19 en titulaciones de ciencias de la salud}

2020). Los autores de este trabajo creemos que la investigación cualitativa puede ser una importante herramienta para describir las nuevas tendencias de la enseñanza superior en CS en el marco de la pandemia del COVID-19, debido a que explican el cómo y por qué se hacen las cosas (Mukhtar y cols., 2020, Khalil y cols., 2020).

Los autores creemos que es un error aplicar de manera masiva un tipo de metodología intentando encorsetar la realidad, intentado demostrar que es un método efectivo, eficaz y seguro. A modo de ejemplo, Vaona y cols (2018) en su reciente metaanálisis, publicada por la iniciativa Cochrane, demuestran como la aplicación de e-learning para la formación de los profesionales de la salud, no es más segura para los pacientes, ni más eficaz para el cambio de comportamientos de los profesionales de la salud, para la adquisición de nuevas habilidades y competencias y conocimientos, respecto a otras modalidades clásicas, como la enseñanza presencial o a través de sesiones teóricas. La realidad es compleja, y con una pandemia mundial (Yang, 2020, Lippi y cols., 2020) es preciso usar enfoques multidisciplinares y multimodales de investigación (Yang, 2020, Lippi y cols., 2020).

Por otro lado, la investigación cualitativa tiene limitaciones, debido a que no es útil para demostrar la eficacia, eficiencia y seguridad de intervenciones (Creswell y Poth, 2018). Este tipo de respuestas las aportan los diseños experimentales y/o ensayos clínicos (Creswell y Poth, 2018, O’Cathain y cols., 2014). La modificación y aplicación de nuevos programas en enseñanza superior en CS, que buscan aumentar y/o mejorar la eficacia respecto a otras modalidades educativas, deben ser analizados a través de estudios experimentales. Justificar el efecto de nuevas modalidades con otro tipo de metodologías no sería adecuado y daría falsos positivos del efecto de esas nuevas metodologías (Vaona y cols., 2018; O’Cathain y cols., 2014). Dentro de las opciones a utilizar, se encuentran los diseños mixtos ( 0 L $H G H H M H F K$ ) que incorporan metodologías cualitativas y cuantitativas simultáneamente en el mismo diseño, y permiten analizar situaciones (Curry y Nunez-Smith, 2015, Tuzzio y cols., 2019). De este modo se podría, investigar el impacto del COVID-19 en los procesos de enseñanza y aprendizaje en la educación superior en profundidad, respondiendo no sólo a el cómo y por qué, sino al cuanto y medir con precisión la efícacia y la efectividad de cambios en el modo de impartir la educación superior (Shehata y cols., 2020, Poncette y cols., 2020). Shehata y cols (2020) describen a través de un diseño mixto, como la pandemia de COVID19 impactó en la enseñanza en las Facultades de Medicina, y su proceso de adaptación. Por otro lado, en Alemania, Poncette y cols (2020) en su estudio mixto, mostraban la capacidad de adquisición de conocimientos tecnológicos de los estudiantes de medicina para adaptarse a la enseñanza a través de modalidad online.

Otro aspecto es la repercusión en los pacientes, que los cambios en la modalidad de enseñanza pueden provocar. Los estudiantes, cuando realizan prácticas clínicas, prácticum, aplican conocimientos y habilidades, en un contexto real sanitario, donde sus acciones repercuten en las personas enfermas. Personas con multitud de enfermedades, madres y padres con sus hijos con enfermedades terminales, cáncer, ingresos en la unidad de cuidados intensivos, y un largo etc, que obliga a los docentes a evaluar y monitorizar estrechamente, si la aplicación de nuevas modalidades de enseñanza permite adquirir competencias necesarias e CS manteniendo la seguridad de las personas enfermas y del estudiante.

La pandemia ha obligado a optimizar recursos, y usar nuevas formas de comunicación y relación con los estudiantes a través de plataformas digitales; Zoom ${ }^{\circledR}$, Microsoft Teams ${ }^{\circledR}$, etc (Khalil y cols., 2020, Jowsey y cols., 2020). Unido a estas nuevas plataformas de comunicación, los materiales docentes también deben adaptarse y cambiar. En esta nueva adaptación los estudiantes deben participar en el diseño e integración de los materiales docentes (Jowsey y cols., 2020). Los autores creemos que existe una oportunidad de poder 
investigar en titulaciones de CS, que material docente es el más adecuado para ser integrado en HODQQJ y de este modo optimizar recursos (Khurshid y cols., 2020, Dewart y cols., 2020).

Los autores creemos que realizar propuestas de investigación basada en preguntas diferentes, es una oportunidad para adaptar la docencia a entornos complejos y cambiantes (Patel y cols., 2020). El análisis de cómo enseñan y aprenden los profesores y estudiantes de CS, como se maneja y utiliza el material docente, como impacta las nuevas modalidades en los estudiantes de CS y en sus docentes, y en el desarrollo de buenas prácticas de uso del material a la hora de impartir asignaturas, ayudará a las titulaciones de CS a adaptarse a este nuevo marco de enseñanza superior.

\section{Conclusiones}

La pandemia del COVID-19 ha provocado utilizar diferentes métodos de enseñanza que ha impactado en los profesores y estudiantes. Los autores de este trabajo, creemos que, en las titulaciones de CS, es preciso analizar el proceso de enseñanza y aprendizaje durante la pandemia, y su influencia en la adquisición de competencias. El cómo se hacen las cosas, y por qué se hacen de una determinada manera, influyen en los resultados.

En futuras investigaciones se demostrará si las adaptaciones realizadas han contribuido positivamente en la enseñanza en titulaciones de CS. Pero es preciso, resaltar que las competencias en CS están integrada con el contacto real con personas enfermas. La pandemia puede ayudar a reflexionar y replantear la integración de nuevas herramientas docentes. Integración de nuevas metodologías y paradigmas en CS, debería ser un proceso evaluado teniendo en cuenta las repercusiones en la persona enferma, debido a que es la receptora de la calidad de la formación que reciben las estudiantes.

\section{Referencias}

ARCHIBALD, M.M., AMBAGTSHEER, R.C., CASEY, M.G y LAWLESS, M. (2019). "Using zoom videoconferencing for qualitative data collection: Perceptions and experiences of researchers and participants" en International Journal of Qualitative Methods, 18.

CAROLAN, C.M., FORBAT, L y SMITH, A. (2016). "Developing the DESCARTE Model: The Design of Case Study Research in Health Care" en Qualitativa Health Research, Vol. 26, issue 5, p.626639.

CRESWELL, J.W y POTH, C.N. (2018). Qualitative inquiry and research design. Choosing among five approaches. Thousand Oaks: Sage. 4th ed.

CURRY, L y NUNEZ-SMITH, M. (2015). Mixed Methods in Health Sciences Research: A Practical Primer. Thousand Oaks, CA: Sage Publications.

DEWART, G., CORCORAN, L., THIRSK, L y PETROVIK, K. (2020). "Nursing education in a pandemic: Academic challenges in response to COVID-19" en Nurse Education Today, vol 92:104471.

DICKINSON, B.L., GIBSON, K., VANDERKOLK, K., GREENE, J., ROSU, C.A., NAVEDO, D.D y cols. (2020). "It is this very knowledge that makes us doctors: an applied thematic analysis of how medical students perceive the relevance of biomedical science knowledge to clinical medicine" en BMC Medical Education, vol. 20, issue 1, p. 356.

GARG, M., ENIASIVAM, A., SATTERFIELD, J., NORTON, B., AUSTIN, E y DOHAN, D. (2020). "Rapid transition of a preclinical health systems science and social justice course to remote learning in the time of coronavirus" en Medical Education Online, vol. 25, issue 1, p. 1812225.

GOBIERNO DE ESPAÑA. Ley Orgánica 15/1999, de 13 de diciembre, de Protección de Datos de Carácter Personal. BOE, 14 de diciembre de 1999. <https://www.boe.es/buscar/act.php?id=BOE-A-1999$23750>$

(c)) BY-NC-ND 2021, Universitat Politècnica de València

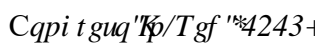




\section{Aplicación de la metodología cualitativa para el estudio del impacto de la pandemia COVID-19 en titulaciones de ciencias de la salud}

GOBIERNO DE ESPAÑA. Ley Orgánica 3/2018, de 5 de diciembre, de Protección de Datos Personales

y garantía de los derechos digitales. BOE, 6 de diciembre de 2018. $<$ https://www.boe.es/buscar/act.php?id=BOE-A-2018-16673\&p=20181206\&tn=2>

JOWSEY, T., FOSTER, G., COOPER-IOELU, P y JACOBS, S. (2020). "Blended learning via distance in pre-registration nursing education: A scoping review" en Nurse Practice Educator, vol. 44, 102775.

KHALIL, R., MANSOUR, A.E., FADDA, W.A., ALMISNID, K., ALDAMEGH, M., AL-NAFEESAH, A y cols. (2020). "The sudden transition to synchronized online learning during the COVID-19 pandemic in Saudi Arabia: a qualitative study exploring medical students' perspectives" en BMC Medical Education, vol. 20, issue 1, p. 285.

KHURSHID Z., DE BRÚN, A., MOORE, G y MCAULIFFE, E. (2020). "Virtual adaptation of traditional healthcare quality improvement training in response to COVID-19: a rapid narrative review", en Human Resources for Health, vol. 18, issue 1, p.81.

KORSTJENS, I y MOSER, A. (2017). "Series: Practical guidance to qualitative research. Part 2: Context, research questions and designs" en European Journal of General Practice, vol. 23, issue 1, p. 274279

LEAVY, P. (2018). + DQCERRNRII\$ UWW\%DHG55 HMDUFK New York, USA: The Guilford Press.

LEVITT, H. M., BAMBERG, M., CRESWELL, J.W., FROST, D.M., JOSSELSON, R y SUÁREZOROZCO, C. (2018). “Journal article reporting standards for qualitative primary, qualitative metaanalytic, and mixed methods research in psychology: The APA Publications and Communications Board task force report" en American Psychologist Journal, vol. 73, issue 1, p. 26-46.

LIPPI, G., SANCHIS-GOMAR, F y HENRY, B.M. (2020). "COVID-19: unravelling the clinical progression of nature's virtually perfect biological weapon" en Annals of Translational Medicine, vol. 8, issue 11, p. 693.

MARTINS, V.S.M., SANTOS, C.M.N.C., BATAGLIA, P.U.R., DUARTE, I.M.R.F. (2020). “The Teaching of Ethics and the Moral Competence of Medical and Nursing Students" en Health Care Analysis. 10.1007/s10728-020-00401-1.

MATUA, G.A y VAN DER WAL, D.M. (2015). "Differentiating between descriptive and interpretive phenomenological research approaches", en Nursing Research, vol. 22, issue 6, p. 22-27.

MBAKAYA, B.C., KALEMBO, F.W., ZGAMBO, M., KONYANI, A., LUNGU, F., TVEIT, B., y cols. (2020). "Nursing and midwifery students' experiences and perception of their clinical learning environment in Malawi: a mixed-method study" en BMC Nursing, vol. 19, p. 87.

MILES, M., HUBERMAN, A y SALDAÑA, J. (2013). Qualitative Data Analysis: A Method Sourcebook, Thousand Oaks: Sage, 3rd ed.

MOSER, A y KORSTJENS, I. (2018). "Series: Practical guidance to qualitative research. Part 3: Sampling, data collection and analysis" en European Journal of General Practice, vol. 24, issue 1, p. 9-18.

MUKHTAR, K., JAVED, K., AROOJ, M y SETHI, A. (2020). "Advantages, Limitations and Recommendations for online learning during COVID-19 pandemic era" en Pakistan Journal of Medical Science, vol. 36(COVID19-S4) p. 27-31.

O'BRIEN, B.C., HARRIS, I.B., BECKMAN, T.J., REED, D.A y COOK, D.A. (2014). "Standards for reporting qualitative research: a synthesis of recommendations" en Academic Medicine, vol. 89, issue 9, p. 1245-1251.

O'CATHAIN, A., THOMAS, K.J., DRABBLE, S., RUDOLPH, A., GOODE, J y HEWISON, J. (2014). "Maximising the value of combining qualitative research and randomised controlled trials in health research: The Qualitative Research in Trials (QUART) study—A mixed methods study" en Health Technology Assess, Vol. 18, issue1. 
PATEL, N.M., KHAJURIA, A y KHAJURIA, A. (2020). "Utility of a webinar to educate trainees on UK core surgical training (CST) selection - A cross sectional study and future implications amidst the COVID-19 pandemic" en Annals of Medicine and Surgery, vol.59, p.35-40.

PATELAROU, A.E., MECHILI, E.A., RUZAFA-MARTINEZ, M., DOLEZEL, J., GOTLIB, J., SKELASAVIC, B y cols. (2020). "Educational Interventions for Teaching Evidence-Based Practice to Undergraduate Nursing Students: A Scoping Review" en International Journal of Environmental Research and Public Health, vol. 17, issue 17, p. 6351.

PONCETTE, A.S., GLAUERT, D.L., MOSCH, L., BRAUNE, K., BALZER, F y BACK, D.A. (2020). "Undergraduate Medical Competencies in Digital Health and Curricular Module Development: Mixed Methods Study" en Journal of Medical Internet Research, vol. 22, issue 10, p. 22161.

SHEHATA M.H., ABOUZEID, E., WASFY, N.F., ABDELAZIZ, A., WELLS, R.L., AHMED, S.A. (2020). "Medical Education Adaptations Post COVID-19: An Egyptian Reflection" en Journal of Medical Education and Curricular Development, vol. 7, p. 1-9.

SNEYD, J.R., MATHOULIN, S.E., O'SULLIVAN, E.P., SO, V.C., ROBERTS, FR, PAUL, A.A y cols. (2020). "Impact of the COVID-19 pandemic on anaesthesia trainees and their training" en British Journal of Anaesthesia, vol. 125, issue 4, p. 450-455.

TENNY, S., BRANNAN, G.D., BRANNAN, J.M y SHARTS-HOPKO, N.C. (2020). Qualitative Study. In: StatPearls. Treasure Island (FL): StatPearls Publishing; July 1

TONG, A., SAINSBURY, P y CRAIG, J. (2007). "Consolidated criteria for reporting qualitative research (COREQ): a 32-item checklist for interviews and focus groups" en International Society for Quality in Health Care, vol. 19, issue 6, p. 349-357.

TUZZIO, L., LARSON, E.B., CHAMBERS, D.A., CORONADO, G.D., CURTIS, L.H., WEBER, W.J y cols. (2019). "Pragmatic clinical trials offer unique opportunities for disseminating, implementing, and sustaining evidence-based practices into clinical care: Proceedings of a workshop" en healthcare Amsterdam, vol. 7, p. 51-57.

VAONA, A., BANZI, R., KWAG, K. H., RIGON, G., CEREDA, D., PECORARO, V y cols. (2018). "(

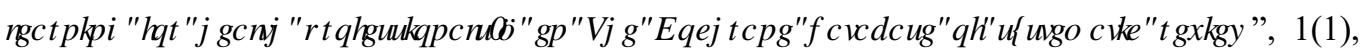
CD011736.

WILSON, A. (2015). "A guide to phenomenological research" en Nursing Standard, vol. 29, issue 34, p. $38-43$.

YANG, W. (2020). "Editorial commentary on special issue of COVID-19 pandemic" en Journal Biomedical Reseach, vol. 34 issue 6, p.395-396.

YIN, R.K. (2014). Case Study Research: Design and Methods. Thousand Oaks, CA: Sage. 5 th ed 